\title{
Adaptive Filters for Muscle Response Suppression
}

\section{Sennels, Søren; Biering-Soerensen, Fin; Hansen, Steffen Duus; Andersen, Ole Trier}

\section{Published in:}

Proceedings of the 18th Annual International Conference of the IEEE Engineering in Medicine and Biology

Link to article, DOI:

10.1109/IEMBS.1996.651844

Publication date:

1996

Document Version

Publisher's PDF, also known as Version of record

Link back to DTU Orbit

\section{Citation (APA):}

Sennels, S., Biering-Soerensen, F., Hansen, S. D., \& Andersen, O. T. (1996). Adaptive Filters for Muscle Response Suppression. In Proceedings of the 18th Annual International Conference of the IEEE Engineering in Medicine and Biology (Vol. Volume 2, pp. 518-520). IEEE. https://doi.org/10.1109/IEMBS.1996.651844

\section{General rights}

Copyright and moral rights for the publications made accessible in the public portal are retained by the authors and/or other copyright owners and it is a condition of accessing publications that users recognise and abide by the legal requirements associated with these rights.

- Users may download and print one copy of any publication from the public portal for the purpose of private study or research.

- You may not further distribute the material or use it for any profit-making activity or commercial gain

- You may freely distribute the URL identifying the publication in the public portal 


\title{
ADAPTIVE FILTERS FOR MUSCLE RESPONSE SUPPRESSION
}

\author{
Søren Sennels, Fin Biering-Sørensen ${ }^{1}$, Steffen Duus Hansen, Ole Trier Andersen ${ }^{2}$ \\ Department of Mathematical Modelling, Build. 349, Technical University of Denmark, \\ DK-2800 Lyngby, Denmark. E-mail: sennels@ei.dtu.dk
}

\begin{abstract}
To be able to use the voluntary EMG-signal from an electrically stimulated muscle as control signal for FES-applications, it is necessary to eliminate the muscle response evoked by the stimulation. 'The muscle response is a non-stationary signal, therefore a set of linear adaptive prediction filters are proposed, based on the observation that the shape of the muscle responses only exhibits moderate changes during a time window of up to $300 \mathrm{~ms}$. The filters are derived and compared with a conventional fixed comb filter on both simulated and real data. For variations in amplitude of the muscle responses the performance of the adaptive filters are independent of the filter length, whereas for variations in the shape the performance is increased with the filter length. Using the adaptive filters it is possible to obtain a signal-to-noise ratio, which enables the EMG from a partly paralysed muscle to be used as control signal for stimulation of the same muscle.
\end{abstract}

Keywords-- Functional Electrical Stimulation, EMGcontrol, Adaptive Filters.

\section{INTRODUCTION}

$\mathrm{T}$ HE EMG-signal has often been used as a control signal in FES-applications, but due to difficulties in extracting the voluntary EMG from a muscle, which is being electrically stimulated, in most cases the EMG from one muscle is used for controlling the stimulation of another muscle [1], [2]. The EMG from an electrically activated muscle consists of both the patient's voluntary neuromuscular activity and artifacts caused by the stimulation, and is essentially non-stationary. Therefore an adaptive filtering approach is proposed. If an effective elimination of the artifacts is carried out, it is possible to use the EMG from a muscle to control the stimulation of the same muscle. This will give the user a more natural control of his/hers neural prosthesis.

\section{METHODS}

When recording the EMG from a muscle, which is being simultaneously electrically stimulated, the measured signal will consist of 3 components: 1) a stimulation artifact, which is evoked by the direct, spill-over between the stimulating and recording electrodes, 2) a muscle response, which is due to the synchronous activation of many motor units, 3) the voluntary EMG, caused by the patient's desire for muscular activity. The artifacts are typically more than an order of magnitude larger than the voluntary EMG, which can be regarded as a band limited white-noise signal. The filtering task is to extract the voluntary EMG

\footnotetext{
${ }^{1}$ Centre for Spinal Cord Injured, University Hospital, Copenhagen
} ${ }^{2}$ Dep. of Information Technology, Tech. University of Denmark.
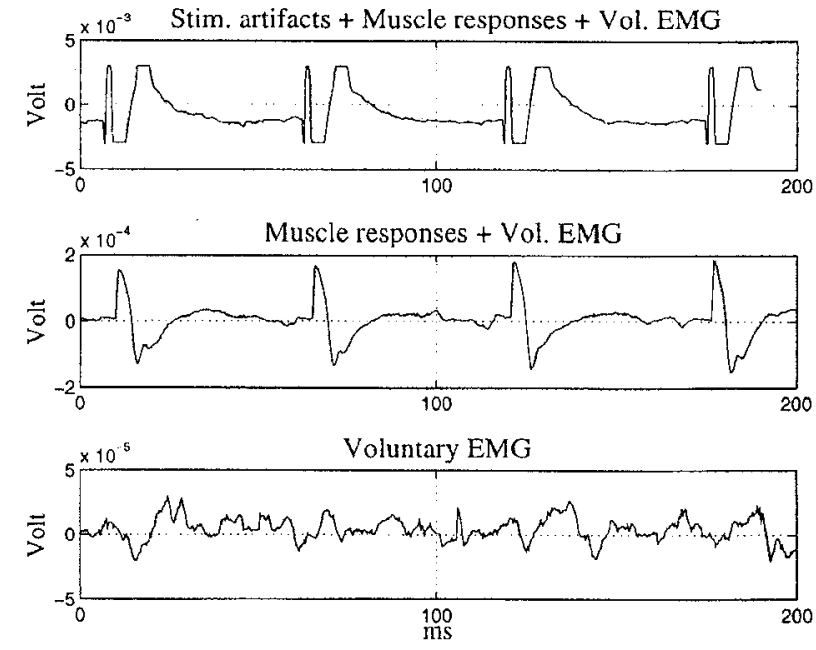

Fig. 1. Example of a recorded signal from a wrist extensor muscle, which is voluntary contracted and stimulated at $20 \mathrm{~Hz}$.

from the recorded signal (fig. 1).

The stimulation artifacts are eliminated by shutting down the amplifier during the stimulation pulses as described previously [3]. This will effectively suppress the stimulation artifacts and leave only the muscle responses and the voluntary EMG (middle in fig. 1).

Since the muscle response in some cases can extend for an entire stimulation period additional filtering is necessary. Some authors have assumed the muscle responses to be stationary and therefore used fixed comb filters [4], [5]. But the muscle response is essentially non-stationary and depends on stimulation amplitude, electrode position, limb position, contraction level etc. Therefore an adaptive linear prediction filter is proposed as shown in fig. 2 , based on the following observation: During a relatively small time interval (typ. $<300 \mathrm{~ms}$ ), consisting of only a few stimulation periods, the amplitude of the muscle responses can exhibit relatively large variations as the stimulation strength is changed, whereas the shape of the response only exhibits small variation, mainly due to limb movements. The idea is to divide the input signal into frames of length $N$, where $\mathrm{N}$ is the ratio between the sampling frequency and stimulation frequency. Each frame then only consist of the muscle response caused by one stimulation pulse. The present frame is predicted on the basis of a linear combination of $\mathrm{M}$ foregoing frames. By subtracting the predicted frame from the actual frame the muscle responses can be 


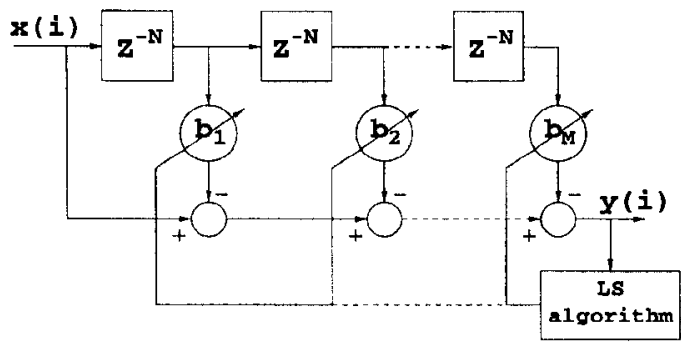

Fig. 2. Linear adaptive prediction filter for suppression of muscle responses.

eliminated, leaving a signal, which after some scaling has the same RMS-value as the voluntary EMG. The output of the filter is given by:

$$
y(i)=x(i)-\sum_{j=1}^{M} b_{j} x(i-j N)
$$

The optimal filter coefficients are found by minimizing the output power by a Least-Sqaure algorithm [6]:

$$
\frac{d}{d b_{j}}\left(\sum_{i=1}^{N} y_{i}^{2}\right)=0, \quad \text { for } \mathrm{j}=1 \ldots \mathrm{M}
$$

Solving (2) gives an usual set of normal equations from which the filter coefficients are derived:

$$
\mathbf{\Phi} \mathbf{b}=\Theta \Leftrightarrow \mathbf{b}=\mathbf{\Phi}^{-1} \Theta
$$

where,

$$
\begin{aligned}
\mathbf{\Phi} & =\left[\begin{array}{cccc}
\phi(1,1) & \phi(1,2) & \cdots & \phi(1, M) \\
\phi(2,1) & \phi(2,2) & \cdots & \phi(2, M) \\
\vdots & \vdots & \ddots & \vdots \\
\phi(M, 1) & \phi(M, 2) & \cdots & \phi(M, M)
\end{array}\right] \\
\boldsymbol{\Theta} & =[\phi(0,1), \phi(0,2), \ldots, \phi(0, M)]^{T} \\
\mathbf{b} & =\left[b_{1}, b_{2}, \ldots, b_{M}\right]^{T} \\
\phi(j, k) & =\sum_{i=1}^{N} x(i-j N) x(i-k N), \text { for } \mathrm{j}, \mathrm{k}=1 \ldots \mathrm{M}
\end{aligned}
$$

(3) is being solved in real time on a ADSP-21020, using LU-decomposition followed by forward- and backsubstitution [7].

\section{RESULTS}

Adaptive filters with $\mathrm{M}=1,3$ and 6 have been evaluated and compared with a fixed filter with $\mathrm{M}=1$ and $b_{1}=1$ on both simulated and real data with $\mathrm{N}=111$ corresponding to a stimulation frequency of $30 \mathrm{~Hz}$ and a sampling frequency of $3.33 \mathrm{kHz}$.

\section{A. Simulated data}

The muscle responses have been simulated as an exponential damped sinus with amplitude, $a$, and time constant, $\tau$, and the voluntary EMG as additive band-limited
Gaussian Noise $\left(\mu=0, \sigma^{2}=1\right)$. The input signal is simulated as:

$$
x(i)=a \cdot e^{-\tau / i} \sin (2 \pi 3 i / N)+n(i), \text { for } \mathrm{i}=1 \ldots \mathrm{N}
$$

where $a=200$ and $\tau=20$ are chosen to give a signal-tonoise ratio of app. $-32 \mathrm{~dB}$ and so the muscle response is significant larger than the noise-signal in the entire stimulation period. This can be regarded as a worst case situation. Both $a$ and $\tau$ were exposed to random variations around the fixed value to simulate differences in amplitude and shape respectively. Both the pure noise signal, $n(i)$, and the mixed signal, $x(i)$, were processed by the filters, and a Filter Performance Index (FPI) was defined as:

$$
\mathrm{FPI}=10 \log \left(\frac{\sum n(i)^{2}}{\sum x(i)^{2}}\right)
$$

and calculated both from $x(i)\left(\mathrm{FPI}_{i}\right)$ and $y(i)\left(\mathrm{FPI}_{\mathrm{O}}\right)$. A perfect filtering, where all of the muscle responses are eliminated, will result in a $\mathrm{FPI}_{\mathrm{O}}$ of $0 \mathrm{~dB}$. Some selected results are shown in table $\mathrm{I}$.

\begin{tabular}{|rc|r|rrrr|}
\hline $\begin{array}{c}\mathrm{a}=200 \\
\pm\end{array}$ & $\tau=20$ & $\mathrm{FPI}_{\mathrm{i}}$ & \multicolumn{4}{|c|}{$\mathrm{FPI}_{\mathrm{O}}[\mathrm{dB}]$} \\
\hline $0 \%$ & $0 \%$ & -32.8 & 0.0 & 0.0 & -0.1 & -0.2 \\
\hline $100 \%$ & $0 \%$ & -33.2 & -28.9 & -5.8 & -1.5 & -0.4 \\
\hline $0 \%$ & $40 \%$ & -33.5 & -17.8 & -11.4 & -0.7 & -0.3 \\
\hline $0 \%$ & $100 \%$ & -32.7 & -28.9 & -19.8 & -4.8 & -2.6 \\
\hline $100 \%$ & $100 \%$ & -33.2 & -30.3 & -17.0 & -7.5 & -2.4 \\
\hline
\end{tabular}

TABLE I. Selected simulated results.

For a signal where the muscle responses are identical in each frame, all of the filters show perfect performance. When adding random variation to the amplitude, the fixed filter performance is drastically reduced, whereas the performance of the adaptive filters are still good, even for very large variations. Variation to the shape of the muscle responses were done by applying random variations to $\tau$. In this case both the fixed and $M=1$ adaptive filter is showing decreasing performance, whereas the $M=3$ and $M=6$ adaptive filters are still having a good performance. Only for very large variations in shape the $M=6$ filter is performing slightly better than the $M=3$ filter. Applying variations in amplitude together with variations in shape does not affect the filter performances further.

\section{B. Real data}

The filters were also evaluated on real muscle responses obtained from the wrist extensor muscles on both healthy and tetraplegic individuals. Since it is not possible to process both the pure voluntary EMG and the muscle responses through the filters, the FPI in (5) cannot be calculated. Instead is the power reduction, PR, defined as:

$$
\mathrm{PR}=10 \log \left(\frac{\sum x(i)^{2}}{\sum y(i)^{2}}\right)
$$


On healthy individuals a set of input signals were generated, with the stimulation applied as a positive or negative ramp, and with elbow flexion, forearm pronation and forearm supination during constant stimulation. On tetraplegic individuals only signals with the stimulation amplitude applied as a ramp were generated. Each filter was applied to the set of input signals and PR was calculated. Table II shows some selected results obtained from real data. Since PR depends on the input signal,- only the filter performances within each input signal should be compared.

For real data the fixed and the $M=1$ filter is performing almost equal, whereas a larger power reduction can be obtained by increasing $M$, indicating that the muscle responses are more effectively suppressed.

\begin{tabular}{|l|rrrr|}
\hline & \multicolumn{4}{|c|}{ PR [dB] } \\
Input signal & Fixed & $\mathrm{M}=1$ & $\mathrm{M}=3$ & $\mathrm{M}=6$ \\
\hline Pos. ramp & 14.2 & 14.4 & 19.2 & 23.7 \\
Neg. ramp & 15.1 & 15.8 & 19.8 & 23.8 \\
Elbow flexion & 7.9 & 7.9 & 10.5 & 13.3 \\
Pronation & 15.6 & 15.8 & 19.7 & 22.3 \\
Supination & 11.5 & 12.1 & 12.8 & 15.0 \\
\hline Pos. ramp (SCI) & 12.9 & 12.9 & 14.4 & 17.6 \\
Neg. ramp (SCI) & 14.3 & 14.7 & 16.0 & 17.6 \\
\hline
\end{tabular}

TABILE II. Selected results from real data.

\section{DISCUSSION}

A filtering sequence for adaptive filters with $M=1$ and $M=6$ is shown fig. 3 for an input signal obtained during pronation of the forearm on a healthy individual. The $\mathrm{M}=1$ filter removes most of the muscle responses, but still some artifacts are left in the beginning of each stimulation period. This is mainly due to the limitation, that the $M=1$ filter has to predict the muscle response on the basis of only one frame. The $M=6$ filter can use up to 6 frames to predict the present frame, and is therefore capable of producing a more reliable prediction. In general the filter performance will increase with the filter length, due to the whitening property of a linear prediction filter.

In section II the EMG-signal was assumed band-limited white noise. This is not entirely correct especially not for a weak muscle activity, where only a few motor units are active. If the number of filter coefficients are chosen to be $M$, but only $p(p<M)$ are necessary to predict the muscle response, the remaining ( $M-p)$ filter coefficients will adapt to the voluntary EMG and therefore reduce the power of the output signal. In a practical case the filter length will be a trade off between a satisfying signal-to-noise ratio and powerful control signal.

\section{CONCLUSION}

An effective elimination of muscle responses are necessary for using the voluntary EMG from a stimulated muscle as a control signal for FES-applications. Due to
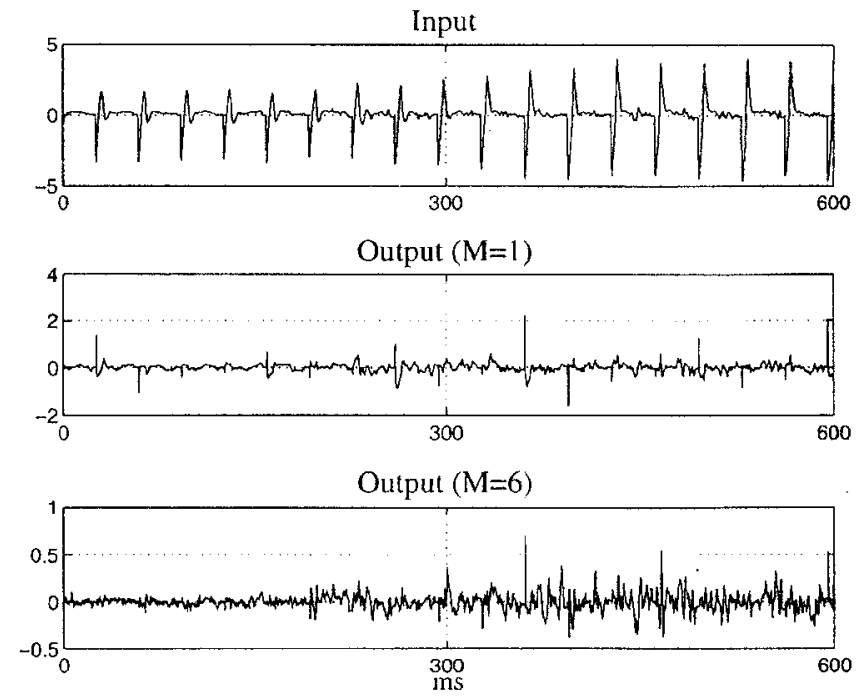

Fig. 3. Example of $M=1$ and $M=6$ filtering of an input signal obtained during forearm pronation.

the non-stationary nature of the muscle response, adaptive filter solutions are useful for establishing a sufficient signal-to-noise ratio. An adaptive linear prediction filter has been proposed, which is capable of eliminating the muscle responses, even during changes in stimulation amplitude and moderate limb movements.

\section{ACKNOWLEDGMENTS}

This project is supported by the Danish Technical Research Council and the Danish Medical Research Council.

\section{REFERENCES}

[1] S. Saxena, S. Nikolić, and D. Popović, "Ax EMG-controlled grasping system for tetraplegics," Journal of Rehabilitation Research and Development, vol. 32, pp. 17-24, February 1995.

[2] P. Peckham, E. Marsolais, and I. T. Mortimer, "Restoration of a key grip and release in the $\mathrm{C} 6$ tetraplegic patient through functional electrical stimulation.," The Journal of Hand Surgery, vol. 5, pp. 462-469, September 1980.

[3] J. Minzly, J. Mizrahi, N. Hakim, and A. Liberson, "Stimulus artefact suppressor for EMG recording during FES by a constantcurrent stimulator.," Medical \& Bioloogical Engineering $\&$ Cornputing, vol. 31 , pp. $72-75,1993$.

[4] H. Holländer, M. Huber, and G. Vossius, "An EMG controlled multichannel stimulator.," in The 9th International Symposium on External Control of Human Extremities, (Belgrade), pp. 291$295,1987$.

[5] E.-U. Haxthausen, Restoration of wrist extcnsion using functional electrical stimulation controlled by the remaining voluntary EMG from the stimulated muscles. PhD thesis, Technical University of Denmark, Electronics Institute, Nov 1992.

[6] S. Haykin, Adaptive Filter Theory. Prentice Hall, 1991.

[7] W. Press, B. Flannery, S. Teukolsky, and W. Vetterling, Numerical Recipies in $C$. Cambridge University Press, 1988. 